\title{
Genes for D-arabinitol and ribitol catabolism from Klebsiella pneumoniae
}

\author{
H. Heuel, A. Shakeri-Garakani, S. Turgut and J. W. Lengeler \\ Author for correspondence: J. W. Lengeler. Tel: +49541969 2290. Fax: +495419692293. \\ e-mail: lengeler@sfbbio1.biologie.uni-osnabrueck.de
}

Universität Osnabrück, Fachbereich Biologie/Chemie, D-49069 Osnabrück, Germany

\begin{abstract}
The enzymes for catabolism of the pentitols D-arabinitol (Dal) and ribitol (Rbt) and the corresponding genes from Klebsiella pneumoniae (dal and rbt) and Escherichia coli (atl and $r t$ ) have been used intensively in experimental evolutionary studies. Four $\mathrm{dal}$ and four $r$ bt genes from the chromosome of $\boldsymbol{K}$. pneumoniae 1033-5P14 were cloned and sequenced. These genes are clustered in two adjacent but divergently transcribed operons and separated by two convergently transcribed repressor genes, dalR and $r b t R$. Each operon encodes an NAD-dependent pentose dehydrogenase (dalD and rbtD), an ATP-dependent pentulose kinase (dalK and $r b t K$ ) and a pentose-specific ion symporter (dalT and rbtn. Although the biochemical reactions which they catalyse are highly similar, the enzymes showed interesting deviations. Thus, DalR (313 aa) and RbtR ( 270 aa) belong to different repressor families, and DalD (455 aa) and RbtD (248 aa), which are active as a monomer or as tetramers, respectively, belong to different dehydrogenase families. Of the two kinases $(19.3 \%$ identity), DalK (487 aa) belongs to the subfamily of short D-xylulokinases and RbtK (D-ribulokinase; 535 aa) to the subfamily of long kinases. The repressor, dehydrogenase and kinase genes did not show extensive similarity beyond local motifs. This contrasts with the ion symporters ( $86.6 \%$ identity) and their genes $(\mathbf{8 2 . 7} \%$ identity). Due to their unusually high similarity, parts of dalT and rbtT have previously been claimed erroneously to correspond to 'inverted repeats' and possible remnants of a 'metabolic transposon' comprising the dal and rbt genes. Other characteristic structures, e.g. a secondary att $\lambda$ site and chi-like sites, as well as the conservation of this gene group in $E$. coli $C$ are also discussed.
\end{abstract}

Keywords: D-arabinitol, ribitol, dal, rbt, Klebsiella pneumoniae, enteric bacteria

\section{INTRODUCTION}

The pentitols ribitol (adonitol; Rbt) and D-arabinitol (Darabitol; Dal) are used by $85 \%$ of Klebsiella strains and by $10 \%$ of Escherichia coli strains, e.g. E. coli C, but not E. coli K-12 and B (Reiner, 1975). Their metabolism in both organisms comprises similar catabolic steps but separate and inducible genes and enzymes. Metabolism starts with an $\mathrm{H}^{+}$symporter for transport and proceeds as summarized in Fig. 1. The corresponding genes are named dal and $r b t$ in Klebsiella, but atl and $r t l$ in E. coli. They are controlled by repressors DalR and RbtR and the inducers $\mathrm{D}$-arabinitol and $\mathrm{D}$-ribulose, respectively (references in Hartley, 1984b; Mortlock, 1984).

The GenBank accession numbers for the sequences reported in this paper are AF045245 (dal) and AF045244 (rbt).
The genetics of pentitol catabolism in enteric bacteria is of interest for several reasons. (i) The dal and $r b t$ genes are adjacent and oppositely oriented, perhaps indicating a gene duplication and inversion which occurred during their evolution (Charnetzky \& Mortlock, 1974c; Neuberger \& Hartley, 1979; Scangos \& Reiner, 1978). (ii) The dal and rbt genes are flanked by long (about $1400 \mathrm{bp}$ ) inverted repeats and are perhaps part of a 'metabolic transposon' (Link \& Reiner, 1982). (iii) The dal and $r b t$ genes are replaced in $\mathrm{Dal}^{-} \mathrm{Rbt}^{-}$strains by the non-homologous gat genes for galactitol metabolism (genotypic exclusion), i.e. these genes map at identical positions but appear to be mutually exclusive (Link \& Reiner, 1983; Reiner, 1975; Woodward \& Charles, 1983). (iv) Pentitol catabolism has been used extensively (for a review see Mortlock, 1982) as a model for studying the acquisition of new metabolic capacities, in particular 


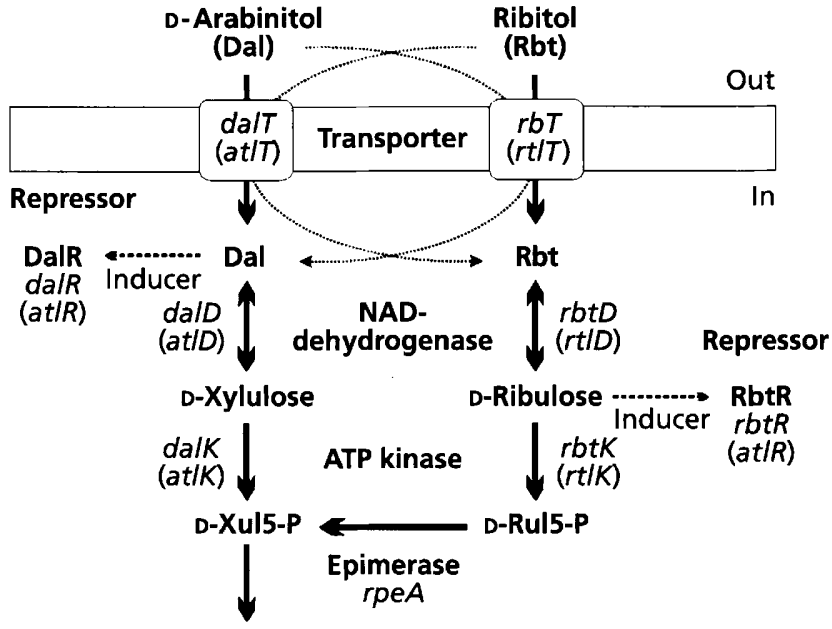

Fig. 1. Metabolic pathways for D-arabinitol and ribitol in enteric bacteria. The metabolic pathways for D-arabinitol (Dal) and ribitol (Rbt) as found in enteric bacteria are shown together with the enzymes and transporters, repressors and inducers, and the corresponding genes. The genes are called $\mathrm{dal}$ and $r b t$ in Klebsiella pneumoniae, but at/ and $r t /$ in $E$. coli. rpeA encoding D-ribulose-5-phosphate epimerase is not one of the dal or rbt genes.

experimental evolution in chemostats involving gene duplications and amplifications (Hartley, 1984a; Mortlock, 1984; Wu et al., 1968).

Despite their importance in evolutionary studies, for most dal and rbt genes only partial sequences are available; neither their exact number nor their nature are known, nor have comparisons for all genes and gene products been presented. Furthermore, contradictory results concerning gene location within the operons of Klebsiella have been published (Charnetzky \& Mortlock, 1974c; Wu et al., 1985). Different nomenclature for the genes of different Klebsiella strains has added to the confusion. In this study we present the complete DNA sequence of the dal and $r b t$ genes from $K$. pneumoniae and describe their location within two operons. We also show that the dal and rbt genes, although encoding enzymes catalysing similar enzymic reactions, are non-homologous, except for the transport systems, and are not flanked by long inverted repeats. Finally, we propose an amended genetic nomenclature for both systems. $r b t B$ and dalB (Charnetzky $\&$ Mortlock, 1974c) are renamed $r b t R$ and dalR (for repressor), $r b t C$ and dalC (Charnetzky \& Mortlock, 1974c) are renamed $r b t D p$ and dalD $p$ (for promoter) and $r t l A B C$ and atlABC (Reiner, 1975) now correspond to $r t D K R$ and atlDKR to comply with official genetic nomenclature.

\section{METHODS}

Bacterial strains, plasmids and culture conditions. Strain KAY2026 (Sprenger \& Lengeler 1984) used in this study as the source of the dal and rbt genes is a phage P1-sensitive $\mathrm{Arg}^{-}$
Gua $^{-}$derivative of Klebsiella pneumoniae (formerly Aerobacter aerogenes) 1033-5P14 (Tanaka et al., 1967). Escherichia coli C (atl rtl), HB101, mutants BL21(2DE3) and JM109 of $E$. coli K-12, and plasmids pSU18, pBluescript II SK $(+$ ) (Ausubel et al., 1990), and pHEX3 and pHEX5 (Heuel et al., 1997) have all been described. Minimal and complex media, and MacConkey indicator plates containing 1\% carbon source have been described previously (Lengeler, 1975).

Transport and enzyme tests. Transport tests using $\left[{ }^{14} \mathrm{C}\right]-$ labelled D-arabinitol, D-mannitol and ribitol were done as described previously taking samples between 0 and $60 \mathrm{~s}$ (Heuel et al., 1997). D-Arabinitol and ribitol dehydrogenases were tested in cell extracts also as described previously (Lengeler \& Lin, 1972). To test the inducibility of the dal and $r b t$ genes, cells were grown exponentially in minimal medium with glycerol as carbon source. For induction, D-arabinitol or ribitol $(0.2 \%)$ were added and the cells were harvested after one further generation. They were washed twice in minimal medium before being tested.

Isolation of plasmid DNA, restriction analysis and cloning procedures. All manipulations with recombinant DNA were carried out by standard procedures as described by Ausubel $e t$ al. (1990). Preparation of plasmid DNA was done by phenol extraction (Sambrook et al., 1989) or by using the JetStar DNA purification system (Genomed), while the isolation of chromosomal DNA from KAY2026 was according to Neumann et al. (1992). Restriction enzymes from various commercial sources were used according to the recommendations of the suppliers. Oligonucleotides for sequencing or PCR were purchased either from TIB Molbiol Syntheselabor or from Life Technologies. DNA amplification by PCR was done according to Saiki et al. (1988) with Taq polymerase from Boehringer Mannheim and an Air ThermoCycler 1605 from Idaho Technology.

DNA sequencing and sequence analysis. To sequence the dal genes cloned into pHHL104 and the rbt genes cloned into pFCK1 (Fig. 2), known restriction sites were used to obtain smaller clones, the ends of which could be sequenced after subcloning. Where needed, longer fragments were shortened by exonuclease III deletion. Finally, specific DNA primers were used to complete the sequences flanking restriction sites. All DNA sequences were determined on both strands by the dideoxy chain-termination method with the T7 sequencing kit from Pharmacia. Computer analysis was done with the GenMon 4.3 program from the Gesellschaft für Biotechnologische Forschung (Braunschweig, Germany) and the BLAST programs and database services provided by the National Center for Biotechnology Information (Bethesda, MD, USA).

T7 RNA polymerase-dependent overexpression and primer extension analysis. T7 RNA polymerase-dependent overexpression of the dal gene products and of $r b t T$ was done according to a protocol described previously (Nobelman \& Lengeler, 1996) using derivatives of medium-copy-number plasmids pHEX3 and pHEX5 (Heuel et al., 1997) and strain BL21( $\lambda$ DE3). The original high-copy-number expression vectors of Tabor \& Richardson (1985) or pBluescript II SK(+) (Ausubel et al., 1990) were exceedingly unstable and could not be used. Primer extension experiments were performed according to Ausubel et al. (1990). To determine the transcription initiation sites of dalD $p$ and dalRp, total cellular RNAs were prepared from HB101(pHHL101) (dalDKT) and from HB101(pHHL102) (dalR). The cells were inoculated into $50 \mathrm{ml}$ of $2 \times$ TY plus chloramphenicol $\left(25 \mu \mathrm{g} \mathrm{l}^{-1}\right)$ to an $\mathrm{OD}_{650}$ of $0 \cdot 1$ and harvested when the cultures reached an 


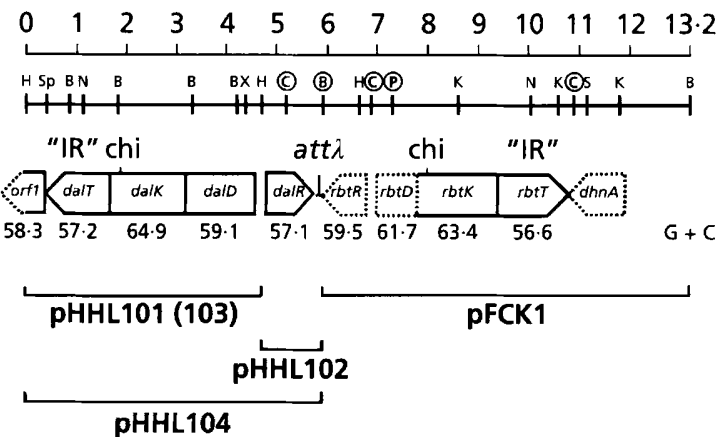

Fig. 2. Structure of the $d a l$ and $r b t$ operons from $K$. pneumoniae. The operons with genes $D$ (dehydrogenase), $K$ (kinase), $T$ (transporter) and $R$ (repressor) were cloned from the chromosome of strain 1033-5P14. The genes are arranged and transcribed (direction of arrows) as indicated. They are flanked by $d h \cap A$, also found in $E$. coli K-12, and an ORF, orf1, of unknown function not found in $E$. coli. dhnA, orf1 and rbtRD sequenced by Hartley (1984b) are indicated by broken arrows. The secondary att $\lambda$ site, the area defined as inverted repeats (IR), chi-like recombination sites (chi), the $\mathrm{G}+\mathrm{C}$ content (mol \%) of the ORFs, a simplified restriction map and essential clones discussed in the text are also shown. The dal genes were cloned in the direction of the lacZp promoter of the vector in pHHL101 but in the opposite direction in $\mathrm{pHHL} 103$. Circled restriction sites are conserved between Klebsiella and $E$. coli C. B, BamHI; C, Clal; H, HindlII; K, Kpnl; N, Ndel; P, Pstl; S, Smal; Sp, Sphl; X, Xhol.

$\mathrm{OD}_{650}$ of $0 \cdot 5$. After centrifugation, the cells were washed, resuspended in $400 \mu \mathrm{l}$ RNA buffer, lysed and treated as described by Ausubel et al. (1990) to isolate total RNAs. Primer extension reactions were also performed according to Ausubel et al. (1990) using a 31 bp primer (see Fig. 4) for both strands. Primers $(50-100 \mathrm{ng})$ were labelled with $\left[\gamma_{-}{ }^{32} \mathrm{P}\right] \mathrm{ATP}$ and T4 polynucleotide kinase. To remove labelled ATP, a 'MicroSpin Sephacryl' column (Pharmacia) was used. A total of 10-50 $\mu \mathrm{g}$ RNA was mixed with $17.5 \mathrm{ng}$ labelled primer and annealed at $65^{\circ} \mathrm{C}$ in hybridization buffer for $90 \mathrm{~min}$. The oligonucleotide primers were extended in the reaction mixture $(45 \mu \mathrm{l})$ containing $5 \mathrm{U}$ avian myeloblastosis virus reverse transcriptase (Boehringer Mannheim) by incubation for $1 \mathrm{~h}$ at $42{ }^{\circ} \mathrm{C}$. After extraction with phenol, precipitation with ethanol and resuspension in $5 \mu \mathrm{l}$ stop solution, according to Ausubel et al. (1990), the cDNAs synthesized were separated by electrophoresis on a $6 \%$ sequencing gel. The size markers were generated by running a dideoxy nucleotide chaintermination reaction with the same primer and plasmid DNA template, including either dalDKT or dalR DNA fragments.

\section{RESULTS AND DISCUSSION}

\section{Cloning of the dal and rbt genes from the chromosome of $K$. pneumoniae}

Previous studies with genes and DNA from two different strains of Klebsiella (Charnetzky \& Mortlock, 1974a, b, c; Hartley, 1984b) and from E. coli C (Link \& Reiner, 1983) indicated that the dal and $r b t$ (atl and $r t l$ ) genes were contiguous. Hartley and co-workers originally transferred the genes from K. pneumoniae 1033-5P14, by means of P1-transduction, into the chromosome of $E$. coli $\mathrm{K}-12$ where the genes inserted at a similar place (near $46 \mathrm{~min}$ ) as in E. coli C (Rigby et al., 1976). Using a secondary att $\lambda$ site near the pentitol-specific genes and a phage $\lambda$ derivative, Neuberger $\&$ Hartley (1979) cloned the dal and $r b t$ genes onto phage $\lambda p r b t$ dal. The phage contained rbtDK and dalDK on a $10.9 \mathrm{kbp}$ insert as identified by growth and enzyme tests; it probably also contained $r b t R$ and dalR because the clones conferred control properties (inducibility) identical to those of parental strain 1033-5P14. The gene order was reported to be dalKD-dalR-rbtR-rbtDK (Fig. 2). This contradicts the order reported by Charnetzky \& Mortlock (1974c) given as dalKD-rbtB-dalB-rbtDK. It is, however, equivalent to the atl rtl gene order as determined by P1transduction for E. coli C (Scangos \& Reiner, 1978).

Because these discrepancies could be due to chromosomal rearrangements that might have occurred during cloning into phage $\lambda$ and because neither all dal (atl) nor all $r b t(r t l)$ genes encoding $\mathrm{D}$-arabinitol and ribitol transport and catabolism had been cloned and identified, we wanted to reclone all genes and map them completely. Hence, chromosomal DNA was isolated from K. pneumoniae KAY2026 (Sprenger \& Lengeler, 1984), one of the strains used by Lin, Hartley and others in their evolutionary studies (references in Mortlock, 1982). The DNA was treated with HindIII before being cloned into the medium-copy-number plasmid pSU18 and transformed into E. coli HB101. This mtlA1 mutant lacks the mannitol-specific phosphotransferase system (II $\left.{ }^{\mathrm{Mtl}}\right)$ as well as the atl and $r t l$ genes and hence the capacity to grow on D-mannitol, D-arabinitol and ribitol. Transformants were first plated on MacConkey indicator plates containing $\mathrm{D}$-arabinitol or $\mathrm{D}$-mannitol. $\mathrm{II}^{\mathrm{Mt1}}$ negative strains can be suppressed to a $\mathrm{Dal}^{+} \mathrm{Mtl}^{+}$ phenotype provided a DalT transporter $($ dalT or atlT), which also transports free D-mannitol, and a Darabinitol dehydrogenase (dalD or atlD), which also converts D-mannitol to D-fructose, are expressed constitutively (Tanaka et al., 1967; Lengeler, 1975; Aulkemeyer et al., 1991; Heuel et al., 1997). Among several thousand transformants containing HindIII DNA fragments, one $\mathrm{Dal}^{+}$(strong) $\mathrm{Mtl}^{+}$(weak) but $\mathrm{Rtl}^{-}$ colony was found. dalT and dalD were subcloned and located on a $4.73 \mathrm{kbp}$ fragment. The fragment was reisolated and religated into the polylinker site of pSU18. The subclones were transformed into strain JM109 and the transformants plated on $\mathrm{LB}_{0}$-Cam plates (Lengeler, 1975), which were supplemented with $1 \mathrm{mM}$ IPTG and $0.02 \%$ X-Gal. From white colonies, plasmids pHHL101 and pHHL103 (Fig. 2) were obtained. Based on their restriction maps, which could be compared to the known maps of dal genes (Hartley, 1984b; Wu et al., 1985; Heuel et al., 1997), it could be concluded that on both plasmids dalD, dalK (encoding a D-xylulose kinase) and dalT had been cloned, but in the opposite direction. After transformation into HB101, all transformants from both plasmids had the same $\mathrm{Dal}^{+} \mathrm{Mtl}^{+}$phenotype. This result implies that the dal genes are expressed constitutively in both clones, i.e. that no DalR-dependent repression occurs. Because in pHHL103 the dal 
Table 1. Gene products of the dal and rbt operons

\begin{tabular}{|c|c|c|c|c|c|c|c|}
\hline $\begin{array}{l}\text { Gene } \\
\text { product }\end{array}$ & Function & No. of aa & $\begin{array}{l}\text { Observed } \\
\text { molecular } \\
\text { mass }(k D a)\end{array}$ & $\begin{array}{c}\text { Calculated } \\
\text { molecular } \\
\text { mass }(\mathrm{Da})\end{array}$ & $\begin{array}{l}\text { Inducer or } \\
\text { major } \\
\text { substrate }\end{array}$ & Type or family & $\begin{array}{c}\text { Identical } \\
\text { residues } \\
(\%)^{*}\end{array}$ \\
\hline $\begin{array}{l}\text { DalR } \\
\text { RbtR }\end{array}$ & Repressor & $\begin{array}{l}313 \\
270\end{array}$ & ${ }^{34 \cdot 9 \dagger}$ & $\begin{array}{l}34940 \\
29700\end{array}$ & $\begin{array}{l}\text { D-Arabinitol } \\
\text { D-Ribulose }\end{array}$ & $\begin{array}{l}\text { DeoR } \\
\text { LacI/GalR }\end{array}$ & None \\
\hline $\begin{array}{l}\text { DaiD } \\
\text { RbtD }\end{array}$ & NAD dehydrogenase & $\begin{array}{l}455 \\
248\end{array}$ & $\begin{array}{l}46 \cdot 5 \dagger \\
27 \cdot 0 \dagger\end{array}$ & $\begin{array}{l}50050 \\
27280\end{array}$ & $\begin{array}{l}\text { D-Arabinitol } \\
\text { Ribitol }\end{array}$ & $\begin{array}{l}\text { Long (monomer) } \| \\
\text { Short (tetramer) }\end{array}$ & None \\
\hline $\begin{array}{l}\text { DalK } \\
\text { RbtK }\end{array}$ & ATP kinase & $\begin{array}{l}487 \\
535\end{array}$ & $\begin{array}{l}54 \cdot 0(110) \ddagger \\
60 \cdot 0(112) \ddagger\end{array}$ & $\begin{array}{l}53570 \\
58850\end{array}$ & $\begin{array}{l}\text { D-Xylulose } \\
\text { D-Ribulose }\end{array}$ & $\begin{array}{l}\text { Hexokinase } \\
\text { Hexokinase }\end{array}$ & $19 \cdot 3$ \\
\hline $\begin{array}{l}\text { DalT } \\
\text { RbtT }\end{array}$ & Transporter & $\begin{array}{l}425 \\
427\end{array}$ & $425^{-}$ & $\begin{array}{l}46750 \\
46970\end{array}$ & $\begin{array}{l}\text { D-Arabinitol } \\
\text { Ribitol }\end{array}$ & $\begin{array}{l}\text { Ion symporter } \\
\text { Ion symporter }\end{array}$ & $86 \cdot 6$ \\
\hline
\end{tabular}

*Local similarities, e.g. for helix-turn-helix motifs or NAD- and ATP-binding motifs, are not considered.

† Purified monomer.

|| Long and short dehydrogenases, according to Persson et al. (1991a, b), active as monomers or as tetramers, respectively.

$\ddagger$ Purified monomer and dimer in parentheses.

§Data from Heuel et al. (1997).

genes have been cloned against the lac $Z p$ promoter of pSU18, the result also implies that the fragment should contain a dalD $p$ promoter (Fig. 2).

In a parallel approach, the $r b t$ genes were isolated after cloning BamHI fragments into the newly constructed medium-copy-number vector pHEX3 (Heuel et al., 1997). Among several thousand HB101 transformants plated on MacConkey ribitol plates was one $\mathrm{Rbt}^{+} \mathrm{Dal}^{-}$ colony. Based on restriction analysis of the new plasmid, pFCK1, a $7.23 \mathrm{~kb}$ BamHI fragment had been cloned (Fig. 2) which, analogous to previous studies (Wu et al., 1985), should contain $r b t R$ (repressor), rbtD (ribitol dehydrogenase) and $r b t K$ (D-ribulose kinase). Again, as for the dal genes, a new promoter-distal gene, $r b t T$, encoding a ribitol-specific ion symporter was also found (Heuel et al., 1997). To clone the missing gene, dalR, which should map between $r b t R$ and dalD (Fig. 2), the terminal sequences of the Bam HI fragment from pFCK1 and of the HindIII fragment from pHHL101 were determined. These were used to devise PCR primers which allowed the synthesis of a DNA fragment carrying dalR flanked by HindIII and BamHI sites using chromosomal DNA as a template. The primers used were primer $1\left(5^{\prime}\right.$ GCCATAGATCACAAAAGAGCAATTACCC $3^{\prime}$ ) starting 31 bp before the HindIII restriction site and primer 2 (5' GGGTTGTGCGCTGGCTGTCGCAGG $3^{\prime}$ ) starting 50 bp after the BamHI restriction site. PCR yielded a $1.2 \mathrm{~kb}$ HindIII-Bam HI fragment which was cloned into pHEX5 to yield pHHL102. The $4.7 \mathrm{~kb}$ HindIII fragment of pHHL101 was isolated next and cloned into the HindIII site of pHHL102. The new plasmid, pHHL104, carried dalRDKT on a $5.9 \mathrm{~kb}$ HindIII-BamHI insert (Fig. 2). The correct insertion of the dal genes on pHHL104 was ascertained by restriction analysis using NdeI, SphI and XboI, three restriction enzymes with single, asymmetrically located cutting sites within the dal genes. Cells of HB101 transformed with pHHL104 exhibited a Dal ${ }^{+}$(strong) phenotype but remained $\mathrm{Mtl}^{-}$. This is the expected phenotype for a dal $R^{+}$strain because $\mathrm{D}$-mannitol is not an inducer for the D-arabinitol-specific DalR repressor. The restriction map of $\lambda p$ rbt dal (Hartley, 1984b) corresponds almost exactly to our map of the $10.93 \mathrm{kbp} H$ indIII-BamHI fragment covering the $r b t$ and dal genes (Fig. 2). Because we isolated the fragment directly from the chromosome of the Klebsiella strain used by these authors, this map should correspond to the genuine map.

\section{Sequencing of the dal and rbt genes from $K$. pneumoniae and identification of their gene products}

We sequenced the entire $10.93 \mathrm{kbp}$ fragment covering the region from orf1 to $d h n A$ completely on both strands, except for $r b t R$ and $r b t D$. First, the sequence data revealed the new genes $d a l T$ and $r b t T$ encoding a Darabinitol- and a ribitol-specific transporter (Heuel et al., 1997), respectively, and second, proved the gene order to be as proposed by Hartley (1984b) and as summarized in Fig. 2. In particular, the dal and $r b t$ genes are adjacent, arranged in a symmetrical but divergent way. The operons are separated by a control region $(2 \cdot 2 \mathrm{kbp})$ encoding the DalR and RbtR repressors. Because dalR and dalDKT are transcribed from opposite strands, and correspondingly for the $r b t$ genes, dalR and $r b t R$ are transcribed convergently as indicated.

Many of the gene products from the dal and $r b t$ genes have been purified and characterized (references in Hartley, 1984b and Wu et al., 1985). The corresponding properties are summarized in Table 1 . The complete peptide sequence for $\mathrm{RbtD}$, and the partial peptide sequences, in particular the amino-terminal sequences, for DalD, DalK and RbtK are available. Furthermore, RbtT (42 kDa) has been made visible in the T7 RNA 
polymerase-dependent overexpression system (Heuel et al., 1997).

\section{DNA sequence analysis and protein comparison}

After complete sequencing of the dal and $r b t$ genes, including the regulatory genes, sequence alignment and comparisons at the DNA and peptide level could be done. Due to previously unresolved DNA compressions, the published sequences had to be corrected in numerous places. These corrections, however, do not alter previous major conclusions. On the other hand, the comparisons revealed some new and unexpected results (Fig. 2 and Table 1).

\section{dal $R$ and $r b t R$ encoding repressors DalR and RbtR}

dalR and $r b t R$ are transcribed convergently from promoters dalR $p$ and $r b t R p$, respectively (Fig. 2). The secondary att $\lambda$ site (nt 5777-5921), used by Hartley and co-workers (Neuberger \& Hartley, 1979) to clone the $d a l$ and $r b t$ genes onto phage $\lambda$, is located within the intergenic sequence of $209 \mathrm{bp}$. The RbtR repressor (270 aa), which recognizes the molecular inducer Dribulose, belongs to the LacI/GalR family. It contains the typical amino-terminal helix-turn-helix motif (aa525 ) and other characteristic motifs in the central and carboxy-terminal parts (Weickert \& Adhya, 1992). The DalR repressor (313 aa), on the other hand, binds Darabinitol as the molecular inducer and belongs to the DeoR repressor family (van Rooijen \& de Vos, 1990). Characteristic members of this group are DeoR (deoxyribose; about $32 \%$ identical residues), GatR (galactitol), GutR (D-glucitol) and SorC (L-sorbose metabolism) in enteric bacteria. All contain a common inducer-binding motif near the carboxy-terminal end and an aminoterminal helix-turn-helix motif (26-45). Neither dalR and $r b t R$ at the DNA level, nor DalR and RbtR at the peptide level showed any significant similarity.

\section{dalD and $r b t D$ encoding D-arabinitol (DalD) and ribitol dehydrogenase (RbtD)}

In both operons, the promoter-proximal genes dalD and $r b t D$ encode pentose-specific and NAD-dependent dehydrogenases. Against expectation, however, neither dalD and $r b t D$ nor the corresponding proteins showed any similarity at the DNA and peptide level beyond local motifs which identified the gene products as members of the dehydrogenase family. Thus, DalD (455 aa) with affinity to D-arabinitol $\left(K_{\mathrm{M}}{ }^{\text {app }} 24 \mathrm{mM}\right)$ and D-mannitol $\left(K_{\mathrm{M}}\right.$ app $\left.70 \mathrm{mM}\right)$, but not to ribitol, xylitol and D-glucitol, belongs to the subfamily of long ( $\geqslant 450 \mathrm{aa}$ ) dehydrogenases (Persson et al., 1991a, b). These contain a typical NAD-binding (aa 9-17) and substrate-binding (205-211) motif also conserved in the D-mannitol-1-phosphate dehydrogenase of, e.g. E. coli K-12. Long dehydrogenases are active as monomers as has also been shown for DalD (Hartley, 1984b). RbtD (248 aa), by contrast, belongs to the subfamily of short dehydrogenases and acts as a tetramer. Substrates are (in decreasing order)

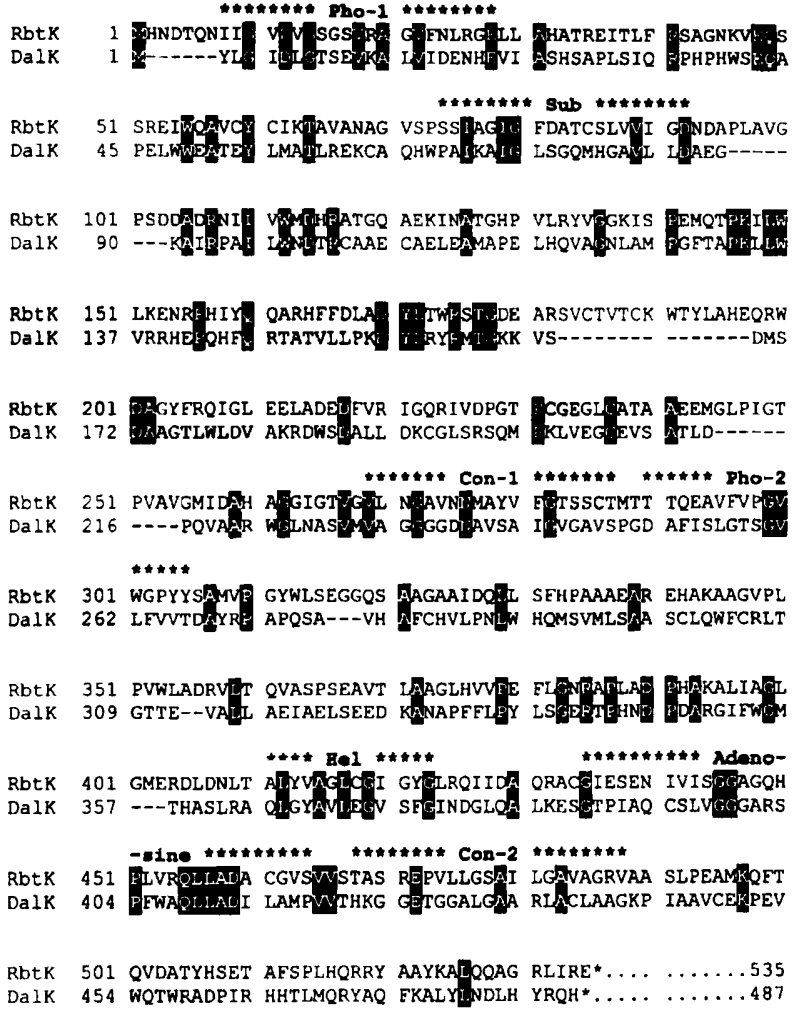

Fig. 3. Comparison of D-xylulose kinase (DalK) and D-ribulose kinase (RbtK). Identical residues (19.3\%) are boxed. The phosphate-binding motifs 1 and 2 (Pho-1/2), the linker or connect motifs 1 and 2 (Con-1/2), the adenosine-binding motif (Adenosine), a helix motif (Hel) and a putative substrate binding area (Sub) are also indicated.

ribitol $\left(K_{\mathrm{M}}{ }^{\text {app }}-11 \mathrm{mM}\right)$, L-arabinitol $(267 \mathrm{mM})$ and xylitol $(1000 \mathrm{mM})$, but not $\mathrm{D}$-arabinitol and D-mannitol (Hartley, 1984b). An NAD-binding motif (21-27) was found as expected.

\section{dalK and rbtK encoding D-xylulose (DaIK) and D- ribulose kinase (RbtK)}

dalK and $r b t K$ encode ATP-dependent kinases for $\mathrm{D}$ xylulose and D-ribulose, respectively (Fig. 3). As for the dehydrogenase genes, dalK and $r b t K$ did not show extended similarities. The D-xylulose kinase DalK (487 aa) is active as a dimer (about $110000 \mathrm{Da}$ ) and resembles the $x y l B$-encoded $\mathrm{D}$-xylulokinase from $K$. pneumoniae (484 aa; $52.9 \%$ identical residues). For comparison, the XylB kinases from $K$. pneumoniae and E. coli share $81.3 \%$ identical residues. Among the many pentoses, pentitols and hexitols, only $\mathrm{D}$-xylulose $\left[K_{\mathrm{M}}\right.$ app $0.8 \mathrm{mM} ; V_{\max } 150 \mu \mathrm{mol} \mathrm{min}^{-1}$ (mg protein) ${ }^{-1}$ ] is a substrate for DalK (Hartley, 1984b). It has been claimed that XylB accepts D-arabinitol and produces Darabinitol 5-phosphate (Scangos \& Reiner, 1979). The three kinases belong to the prokaryotic D-xylulokinase group of the hexokinase family as defined by Bork et al. (1993). Based on three-dimensional crystal structures, hexokinases comprise characteristic structures : in DalK 
(a)

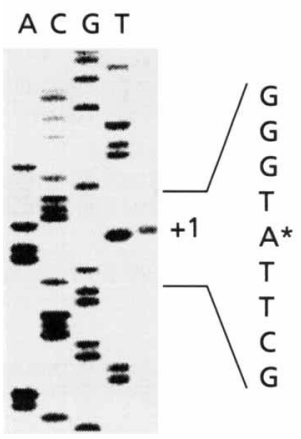

(b)

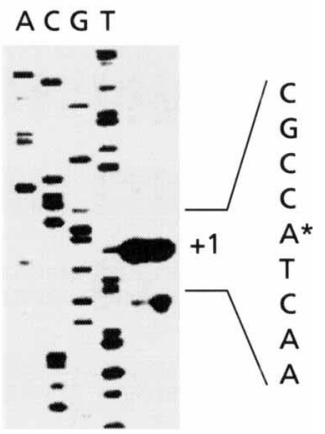

Fig. 4. Mapping of the transcription initiation sites of daIR (a) and dalD (b) by primer extension analysis. Radiolabelled primers complementary to the $5^{\prime}$ end of the non-coding strand of dalR ( $5^{\prime}$ CCATGCGGCACGCACCTTCTGATCCAACCGG 3 ') or dalD ( $5^{\prime}$ CCACGCCTGATGCGCGCGATGAAAAGAACCC 3') were used to direct CDNA synthesis by reverse transcription from total RNAs of cells of strain HB101 containing either pHHL101 (daIDKT) or pHHL102 (da/R). The respective extended primers $(+1)$ are shown in the right-hand lanes. The sequence of the complementary DNA is also given. This was derived from lanes $A, C, G$ and $T$ showing the sequencing ladders with primers labelled at 5 positions by T4 polynucleotide kinase. An asterisk indicates the initiation site, namely an $A$ residue, $50 \mathrm{bp}$ upstream of the putative ATG codon of dalR and $21 \mathrm{bp}$ upstream of the putative ATG codon of daID.

(Fig. 3) these correspond to two phosphate-binding sites (aa 2-22 and 251-266) and an adenosine binding site (388-417), all three involved in ATP binding, two connect or linker structures (230-248 and 421-441) and a putative substrate-binding site (68-86).

The kinase encoded by $r b t K(535 \mathrm{aa})$ also acts as a dimer (about $112000 \mathrm{Da})$. Substrates are D-ribulose $\left[K_{\mathrm{M}}{ }^{\text {app }}\right.$ $0.4 \mathrm{mM} ; V_{\max } 71 \mu \mathrm{mol} \mathrm{min}^{-1}$ (mg protein) ${ }^{-1}$ ] and to a lesser extent $\mathrm{D}$-arabinitol and ribitol $\left(K_{\mathrm{M}}{ }^{\text {app }} 140\right.$ and $220 \mathrm{mM}$, respectively) but not D-xylulose (Hartley, 1984b). Based on sequence alignments, RbtK corresponds to a typical prokaryotic hexokinase, in particular to the group of L-ribulose-, D-gluconate- and glycerol kinases (Bork et al., 1993). Curiously, its closest relative is the hydrophilic part of a large (715 aa) and allegedly membrane-bound protein from Saccharomyces cerevisiae (accession no. Z48785). RbtK shares 25-53\% identical residues with the kinases, the characteristic phosphate 1 and 2 sites (aa 8-28 and 290-305), the connect 1 and 2 motifs (269-287 and 468-488), and the adenosine-binding site (435-464), the latter preceded by a helix motif (411-423) (Fig. 3). The total DalK and RbtK molecules share $19 \cdot 3 \%$ identical residues.

\section{Operons dal and $r b t$ are not flanked by inverted repeats}

It has been claimed repeatedly that $d a l$ and $r b t$ genes are sandwiched between rather long $(0.4-1.4 \mathrm{kbp})$ inverted repeats. It has been speculated that these could be the remnants of a metabolic transposon (Hartley, 1984b)

and perhaps could be involved in genotypic exclusion between the atl and $r t l$ genes and the gat genes in E. coli (Link \& Reiner, 1982). We have shown recently (Heuel et al., 1997) that the dal and $r b t$ operons of $K$. pneumoniae contain previously unidentified genes, dal $T$ and $r b t T$ that are located proximal to genes dalK and $r b t K$, respectively (Fig. 2). The genes encode $\mathrm{D}^{-}$ arabinitol- and ribitol-specific transporters of unusually high similarity $(86.6 \%$ identical amino acids) that is reflected at the DNA level ( $82.7 \%$ identical bases). The postulated inverted repeats correspond to parts of both genes (see IR in Fig. 2), but not to short inverted repeats as they are characteristic of transposable elements. The pronounced similarity starts exactly $6 \mathrm{bp}$ before the ribosome-binding site of dalT and $r b t T$ within intergenic sequences which otherwise did not show any similarity. It ends abruptly within a TTC codon for dalT and a TTT codon for rbtT located 42 and 48 bp before the corresponding stop codons, hence 14 and 16 aa before the end of the peptides, respectively. These residues are parts of the terminal cytoplasmic loop of the permeases. At $58 \mathrm{bp}$ downstream from $r b t T, d b n A$ begins (Fig. 2), a gene found in all atl rtl (dal rbt) and gat strains of $E$. coli and K. pneumoniae tested thus far (unpublished results). dalT on the other side is followed by an ORF not present on the chromosome of E. coli K12.

\section{Regulatory sequences involved in transcription control and size of the complete dal and rbt operons}

Sequence alignments have revealed putative promoter, CRP-binding and ribosome-binding motifs as well as putative initiation and stop codons. DalD, DalK, RbtK and $\mathrm{RbtD}$ have been purified to homogeneity. Their amino-terminal sequences have been used to define putative ribosome-binding sites and initiation codons (references in Hartley, 1984b; Wu et al., 1985).

In the case of the dalR $p$ and the dalD $p$ promoters, the transcription initiation bases were determined directly by primer extension analysis as described in Methods and in the legend to Fig. 4. These are an A residue located 50 bp upstream of the dalR initiation codon and an A residue located $21 \mathrm{bp}$ upstream of the dalD initiation codon. Promoter dalD $p$ contains typical -10 (TACAGT) and -35 (TTATTT) sequences, the latter preceded immediately by a CRP-binding consensus motif (TGTGA $\mathrm{N}_{6}$ GCTCT) with an imperfect symmetry (Fig. 5). Four short palindromes (AATTA) interspersed between these sequences are possibly involved in DalR binding as operators. Similar structures are not found in front of dalR and in dalR $p$. Consistent with these results it was observed that the dal operon in KAY2026 was inducible by external Darabinitol as tested by DalT activity [1 and $46 \mathrm{nmol}$ transport $\min ^{-1}$ (mg protein $)^{-1}$ for uninduced and induced cells, respectively] and that deletion of dalR produced fully constitutive clones [16 nmol transport $\min ^{-1}$ (mg protein $)^{-1}$ for uninduced cells]. Induction was repressed in the presence of glucose (Knott, 1982). 


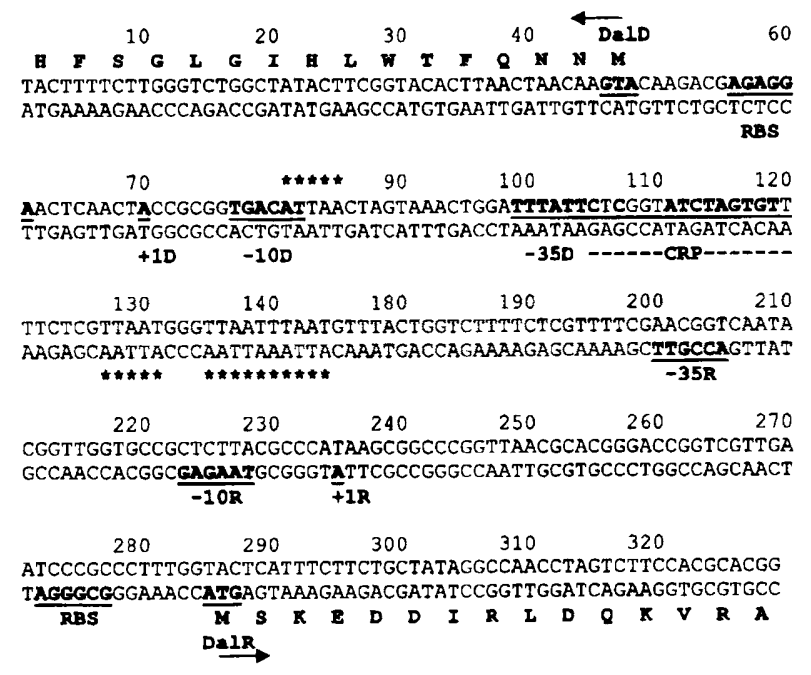

Fig. 5. Regulatory sequences involved in dal gene control. The intergenic sequences located between $d a l D$ and $d a l R$ are shown. Underlined (under the non-coding strand) and in bold type are the initiation codons, transcription initiation sites $(+1)$, as well as putative -10 and -35 binding sites for promoters daIDp (labelled D) and dalRp (labelled R). For dalDp, a putative CRP-binding site (CRP) and short palindromes (asterisks), which may correspond to operator sites, are also shown.

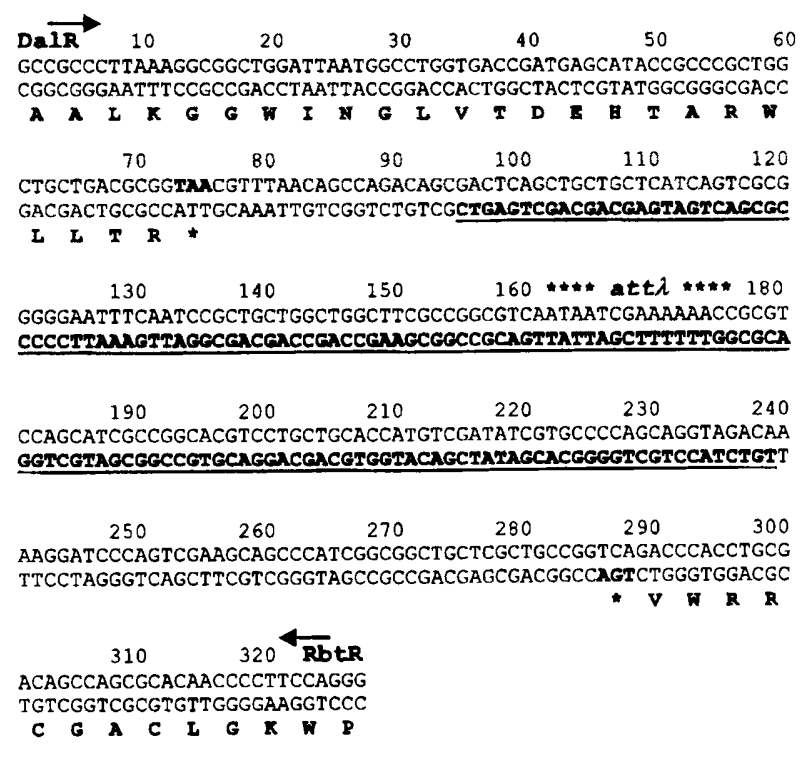

Fig. 6. Intergenic sequences separating the $d a l$ and rbt genes. The ends of dalR and $r b t R$ are shown together with the deduced peptide sequences and stop codons. dal $R$ and $r b t R$ are separated by $209 \mathrm{bp}$. This includes a secondary attachment site for phage $\lambda$ (underlined sequence, att $\lambda$ ), the core of which is indicated (asterisks).

dal $T$ is followed by a short intergenic sequence ( $48 \mathrm{bp}$ ) and an ORF (orf1) of about $1 \mathrm{kbp}$ length. orf1 did not show similarity to any sequence in the sequence databases. Deletion of orf1, as a HindIII-SphI fragment from pHHL101 (Fig. 2), caused no visible change in the Dal phenotype of transformants and preliminary Northern hybridization studies gave no indication for the presence of orf1-specific mRNA in pHHL101 transformants. These results seem to rule out orf1 as a member of the dalDKT operon.

Repressors DalR and RbtR showed no similarity. In accordance with this result, there was no sequence similarity between the intergenic sequences separating $d a l R$ and $d a l D$, and $r b t R$ and $r b t D$, respectively, that carry the corresponding repressor-binding sites. Putative -10 and -35 consensus sequences for promoters $r b t D p$ and $r b t R p$ have been postulated (Wu et al., 1985). Neither these nor a putative CRP-binding sequence(s) in front of $r b t D p$ have been identified and characterized by direct tests. The expression of the $r b t$ genes is, however, strongly reduced during growth on glucose (Neuberger \& Hartley, 1979). Early attempts to follow $r b t D$-specific mRNA synthesis by identifying polysomes engaged in $\mathrm{RbtD}$ synthesis through $\mathrm{RbtD}$-specific antibodies, indicated a dramatic shift in the mid-exponential phase of growth, i.e. a fivefold increase in RbtD activity paralleled by an increase in corresponding polysomes (Hartley, 1984b). It remains to be shown which mechanism underlies this pattern.

\section{Conclusions}

As stated above, the dal and $r b t$ genes have been considered as models for the natural evolution of new operons and metabolic pathways by gene duplication and for metabolic transposons. The present results corroborate previous doubts voiced by Hartley (1984b) on this too simplistic view. Thus, dalR, $D$ and $K$ do not share extensive similarity with $r b t R, D$ and $K$, respectively, and the corresponding repressors, dehydrogenases and kinases belong to different protein families. This argues against evolution by gene or operon duplication followed by diversification and argues for modular evolution ('gene swapping') in which the various genes have been assembled in a mosaic-like way into their present positions. The only area of extensive similarity covers dalT and $r b t T$. Similarity is so high $(82.7 \%$ identical bases and $86.6 \%$ identical amino acid residues) over the entire length of the genes/peptides that it almost certainly indicates a recent origin from a common ancestor, i.e. true homology. It is these DNAs (Fig. 2) which have been considered previously as inverted repeats and the remnants of a putative metabolic transposon (Link \& Reiner, 1982, 1983; Neuberger \& Hartley, 1979).

The evolution of the catabolic pathways for D-arabinitol and ribitol must have been closely linked among the enteric bacteria. Thus, based on biochemical studies, both catabolic pathways are highly similar in Klebsiella (Fig. 1) and in E. coli C (Reiner, 1975), and based on genetic studies the operons are very similar in both organisms and are located at a similar place in the chromosome (Scangos \& Reiner, 1979). We cloned the atl and $r t l$ genes from $E$. coli $\mathrm{C}$ and were able to show that they map in both organisms at an identical location, 
that is between the attP2I site and the $d b n A$ gene (Fig. 2). This location may explain why Rigby et al. (1976) were able to transduce the $d a l$ and $r b t$ genes from $K$. pneumoniae to $E$. coli $\mathrm{K}-12$ by means of phage $\mathrm{P} 1$. Partial sequencing of the atl and $r t l$ genes revealed the expected high similarity with the dal and $r b t$ genes (about $82 \%$ identical bases) and the same operon structure (unpublished results). We were also able to confirm the presence of a secondary attachment site for phage $\lambda$ located between the dal and rbt genes (Fig. 6), which allowed the original cloning of these genes into phage $\lambda$ derivatives (Neuberger \& Hartley, 1979). Finally, we were able to confirm the presence of chi-like recombination sites (GCCTGCC) within dalK (nt 19211927) and rbtK (7974-7980) (see also Fig. 2). These apparently became active in the selection of strains overproducing RbtD during continuous culture on xylitol and caused amplification of dalDR and $r b t R D$ (Neuberger \& Hartley, 1979). These results strongly indicate the presence of a natural mechanism for interspecies horizontal gene transfer of the pentitolspecific genes and enzymes within enteric bacteria.

\section{ACKNOWLEDGEMENTS}

We thank E. Placke for help in preparing the manuscript. For financial support through SFB171 (H.H. and S.T.) and a Graduierten-stipendium (A.S.-G.) we thank the Deutsche Forschungsgemeinschaft and the Verband der Chemischen Industrie for general financial support.

\section{REFERENCES}

Aulkemeyer, P., Ebner, R., Heilenmann, G., Jahreis, K., Schmid, K., Wrieden, S. \& Lengeler, J. W. (1991). Molecular analysis of two fructokinases involved in sucrose metabolism of enteric bacteria. Mol Microbiol 5, 2913-2922.

Ausubel, F. M., Brent, R., Kingston, R. E., Moore, D. D., Seidman, J. G., Smith, J. A. \& Struhl, K. (1990). Current Protocols in Molecular Biology. Chichester: Wiley.

Bork, P., Sander, C. \& Valencia, A. (1993). Convergent evolution of similar enzymatic function on different protein folds: the hexokinase, ribokinase and galactokinase families of sugar kinases. Protein Sci 2, 31-40.

Charnetzky, W. T. \& Mortlock, R. P. (1974a). Ribitol catabolic pathway in Klebsiella aerogenes. J Bacteriol 119, 162-169.

Charnetzky, W. T. \& Mortlock, R. P. (1974b). D-Arabitol catabolic pathway in Klebsiella aerogenes. J Bacteriol 119, 170-175.

Charnetzky, W. T. \& Mortlock, R. P. (1974c). Close genetic linkage of the determinants of the ribitol and D-arabitol pathways in Klebsiella aerogenes. J Bacteriol 119, 176-182.

Hartley, B. S. (1984a). Experimental evolution of ribitol dehydrogenase. In Microorganisms as Model Systems for Studying Evolution, pp. 23-54. Edited by R. P. Mortlock. New York: Plenum Press.

Hartley, B. S. (1984b). The structure and control of the pentitol operons. In Microorganisms as Model Systems for Studying Evolution, pp. 55-107. Edited by R. P. Mortlock. New York: Plenum Press.

Heuel, H., Turgut, S., Schmid, K. \& Lengeler, J. W. (1997). Substrate recognition domains as revealed by active hybrids between the $\mathrm{D}$ arabinitol and ribitol transporters from Klebsiella pneumoniae. J Bacteriol 179, 6014-6019.
Knott, T. J. (1982). The D-arabitol operon of Klebsiella aerogenes. $\mathrm{PhD}$ thesis, University of London.

Lengeler, J. W. (1975). Nature and properties of hexitol transport systems in Escherichia coli. J Bacteriol 124, 39-47.

Lengeler, J. W. \& Lin, E. C. C. (1972). Reversal of the mannitolsorbitol diauxie in Escherichia coli. J Bacteriol 112, 840-848.

Link, C. D. \& Reiner, A. M. (1982). Inverted repeats surround the ribitol-arabitol genes of E. coli C. Nature 298, 94-96.

Link, C. D. \& Reiner, A. M. (1983). Genotypic exclusion: a novel relationship between the ribitol-arabitol and galactitol genes of E. coli. Mol Gen Genet 189, 337-339.

Mortlock, R. P. (1982). Metabolic acquisitions through laboratory selection. Annu Rev Microbiol 36, 259-284.

Mortlock, R. P. (1984). The utilization of pentitols in studies of the evolution of enzyme pathways. In Microorganisms as Model Systems for Studying Evolution, pp. 1-21. Edited by R. P. Mortlock. New York: Plenum Press.

Neuberger, M. S. \& Hartley, B. S. (1979). Investigations into the $K$. aerogenes pentitol operons using specialised transducing phages $\lambda_{\text {p rbt }}$ and $\lambda_{\text {p rbt dal }} . J$ Mol Biol 132, 435-470.

Neumann, B., Pospiech, A. \& Schairer, H. U. (1992). Rapid isolation of genomic DNA from Gram-negative bacteria. Trends Genet 8, 332-333.

Nobelmann, B. \& Lengeler, J. W. (1996). Molecular analysis of the gat genes from Escherichia coli and of their roles in galactitol transport and metabolism. J Bacteriol 178, 6790-6795.

Persson, B., Jeffery, B. \& Jörnvall, H. (1991a). Different segment similarities in long-chain dehydrogenases. Biochem Biophys Res Commun 177, 218-233.

Persson, B., Krook, M. \& Jörnvall, H. (1991b). Characteristics of short-chain alcohol dehydrogenases and related enzymes. Eur J Biochem 200, 537-543.

Reiner, A. M. (1975). Genes for ribitol and D-arabitol catabolism in Escherichia coli: their loci in Escherichia coli $\mathrm{C}$ and absence in K-12 and B strains. J Bacteriol 123, 530-536.

Rigby, P. W. J., Gething, M. J. \& Hartley, B. S. (1976). Construction of intergeneric hybrids using bacteriophage P1CM : transfer of the Klebsiella aerogenes ribitol dehydrogenase gene to Escherichia coli. J Bacteriol 125, 728-738.

van Rooijen, R. J. \& de Vos, W. M. (1990). Molecular cloning, transcriptional analysis and nucleotide sequence of $l a c R$, a gene encoding the repressor of the lactose phosphotransferase system of Lactococcus lactis. J Biol Chem 265, 18499-18503.

Saiki, R. S., Gelfand, D. H., Stoffel, S., Scharf, S. J., Higuchi, R., Horn, G. T., Mullis, K. B. \& Erlich, H. A. (1988). Primer-directed enzymatic amplification of DNA with a thermostable DNA polymerase. Science 239, 487-491.

Sambrook, J., Fritsch, E. F. \& Maniatis, T. (1989). Molecular Cloning: a Laboratory Manual, 2nd edn. Cold Spring Harbor, NY: Cold Spring Harbor Laboratory.

Scangos, G. A. \& Reiner, A. M. (1978). Ribitol and D-arabitol catabolism in Escherichia coli. J Bacteriol 134, 492-500.

Scangos, G. A. \& Reiner, A. M. (1979). A unique pattern of toxic synthesis in pentitol catabolism: implications for evolution. J Mol Evol 12, 189-195.

Sprenger, G. A. \& Lengeler, J. W. (1984). L-Sorbose metabolism in Klebsiella pneumoniae and $\mathrm{Sor}^{+}$derivatives of Escherichia coli K12 and chemotaxis towards sorbose. J Bacteriol 157, 39-45.

Tabor, S. \& Richardson, C. C. (1985). A bacteriophage T7 RNA polymerase/promotor system for controlled exclusive expression of specific genes. Proc Natl Acad Sci USA 82, 1074-1078. 
Tanaka, S., Lerner, S. A. \& Lin, E. C. C. (1967). Replacement of a phosphoenol-pyruvate-dependent phosphotransferase by a nicotinamide adenine dinucleotide linked dehydrogenase for the utilization of mannitol. J Bacteriol 93, 642-648.

Weickert, M. J. \& Adhya, S. (1992). Family of bacterial regulators homologous to Gal and Lac repressors. J Biol Chem 267, 15869-15874.

Woodward, M. J. \& Charles, H.P. (1983). Polymorphism in Escherichia coli: rtl atl and gat regions behave as chromosomal alternatives. J Gen Microbiol 129, 75-84.
Wu, J. C., Anderton Loviny, T., Smith, C. A. \& Hartley, B. S. (1985). Structure of wild-type and mutant repressors and of the control region of the rbt operon of Klebsiella aerogenes. EMBO J 4, 1339-1344.

Wu, T. T., Lin, E. C. C. \& Tanaka, S. (1968). Mutants of Aerobacter aerogenes capable of utilizing xylitol as a novel source of carbon. J Bacteriol 96, 447-456.

Received 20 November 1997; revised 12 February 1998; accepted 16 February 1998. 